Saudi Journal of Oral and Dental Research

Abbreviated Key Title: Saudi J Oral Dent Res

ISSN 2518-1300 (Print) |ISSN 2518-1297 (Online)

Scholars Middle East Publishers, Dubai, United Arab Emirates

Journal homepage: https://saudijournals.com

Review Article

\title{
Teledentistry \& COVID 19 in Tele- Era
}

\author{
Surbhi Priyadarshi ${ }^{1^{*}}$, Pradeep Tangade ${ }^{2}$, Vikas Singh ${ }^{3}$, Najmus Sahar ${ }^{1}$, Debashis Roy ${ }^{1}$ \\ ${ }^{1}$ Postgraduate Student, Department of Public Health Dentistry, Teerthanker Mahaveer Dental College \& Research Centre, Moradabad, \\ Uttar Pradesh, India \\ ${ }^{2}$ Professor \& H. O. D, Department of Public Health Dentistry, Teerthanker Mahaveer Dental College \& Research Centre, Moradabad, \\ Uttar Pradesh, India \\ ${ }^{3}$ Reader, Department of Public Health Dentistry, Teerthanker Mahaveer Dental College \& Research Centre, Moradabad, Uttar Pradesh, \\ India
}

*Corresponding author: Surbhi Priyadarshi

\section{Abstract}

The Covid-19 pandemic has affected the general population at different levels. In the context "prevention is better than cure" certain norms has been implemented by the government like maintaining social distancing and wearing masks. In this pandemic, individuals are facing numerous issues due to minimal availability to physical health and dental care. The solution to this problem of physical unavailability is telemedicine and teledentistry. Teledentistry is a means of virtual communication between patients and consultants which must be incorporated in general healthcare and dental care systems. Though with numerous advantages, this technology also posseses some disadvantages. Proper knowledge and application of this technology should be understood by the healthcare workers for it's suitable implementation. If not fully replace, at least teledentistry can complement the existing compromised dental system during the current pandemic.

Keywords: Teledentistry, telemedicine, COVID-19, pandemic, teletriage, teleconsultations.

Copyright (C) 2021 The Author(s): This is an open-access article distributed under the terms of the Creative Commons Attribution 4.0 International License (CC BY-NC 4.0) which permits unrestricted use, distribution, and reproduction in any medium for non-commercial use provided the original author and source are credited.

\section{INTRODUCTION}

The Covid-19 pandemic has effected the general population at different levels. Due to rapid spread of Covid-19 across the world, entire human population has to face complete lockdown and stay in quarantine. As the transmission of Covid-19 virus is believed to be from one person to others by means of droplets or aerosols, it is of great risk for dentists to interact with their patients physically. Dental health care personnel (DHCP) are categorized under high exposure risk group as stated by Occupational Safety and Health Administration (OSHA), the reason being dentists work in close contiguity to the patient's oral cavity [1]. In many dental procedures like oral prophylaxis, use of rotary instruments aerosols are generated. So to restrict the transmission of aerosols certain protocols should be followed and use of teledentistry should be encouraged.

\section{WHAT IS TELEDENTISTRY?}

Like telemedicine, teledentistry is making the use of informative technology instead of direct face to face contact for the purpose of screening, diagnosing, guiding, educating or treating patients. In 1994, US
Military started the concept of teledentistry to provide service to US troops all around the world [2].

The word 'tele' signifies 'distant' and henceforth fulfills the requirement of maintaining social distancing which is the prime goal to avoid getting infected with Covid-19 Virus. In the ongoing era of Covid-19 pandemic, which may soon be progressed into an endemic, the main aim is to maintain social distancing and to avoid individual to individual contact.

Telehealth should be encouraged as it plays an important role in maintaining distance with individuals or patients. Teledentistry is a type of telehealth utilizing the communication media to consult the patients and to gather the clinical findings of the patients. This concept is also feasible for the patients residing in the outskirts of city with least approach to the dental clinics or hospitals. There are various medium like electronic apps, digital images, webcam-based computers, smartphones, whatsapp etc. for virtual communication between patients and dentists. To prevail over the existing pandemic the significant role of teledentistry in providing dental care services cannot be ruled out. Figure-1 depicts the subunits of teledentistry. 
Surbhi Priyadarshi et al; Saudi J Oral Dent Res, Apr, 2021; 6(4): 157-159

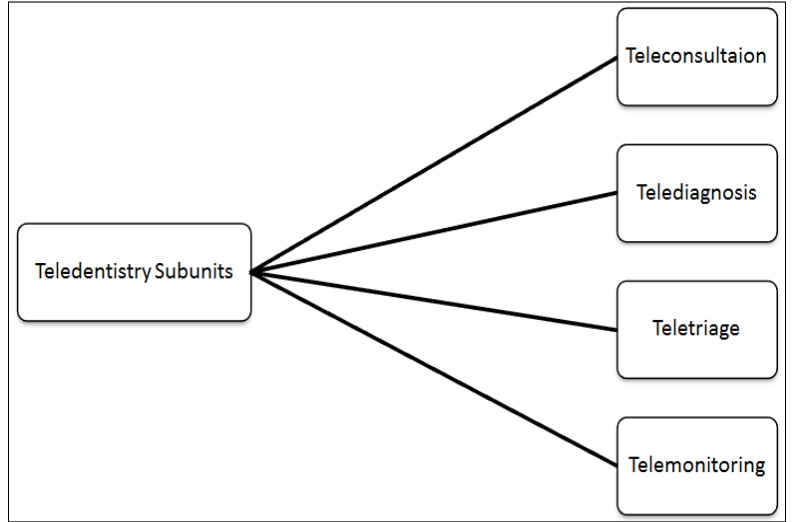

Fig-1: Teledentistry Subunits

\section{TELECONSULTATION}

Teleconsultation is basically telecommunication or use of electromagnetic systems for the purpose of sharing information or communication between patients and dentists. Consulting the patients with physical and intellectual disabilities has been made easy with this type of consultation. It has also been useful in old-aged patients and prisoned individuals [3, 4]. During the period of quarantine and lockdown, teleconsultation has been a boon for general population. Not only this, but it also saves the time required to travel to a dental practitioner.

\section{TELEDIAGNOSIS}

Telediagnosis as the terms suggests the diagnosis or the investigations are made on the basis of pictures and information shared to the dentist with the help of technology. For the diagnosis of dental caries smartphones are recommended [5, 6]. In addition to this, dental lesions which are potentially malignant can also be investigated using Whatsapp and mobile phones. Mobile Mouth Screening Anywhere (MeMoSA®) was introduced by Haron et al. for the purpose of diagnosing oral cancer and concluded it to be advantageous for patients with limited access to specialists [7]. In this pandemic telediagnosis should be used and encouraged to avoid close contacts with the patients.

\section{TELETRIAGE}

In simple words, teletriage means referring the patient to required place for the required treatment at appropriate time. The disposition of the call or the patient to meet the urgent needs is the basic step in teletriage. The aim of the telephone triage is to provide the safest possible treatment in definite period of time. A well trained telephone triage nurses should be appointed for this purpose. Patient's medical/ dental history and his concerned problems are assessed through conversations over telephone and decided whether it can be managed over phone calls or not. It is basically practiced for better accessibility and decreasing the waiting time.

\section{TELEMONITORING}

Generally, patients visit the dental practitioners for the purpose of monitoring. Use of telemonitoring can eliminate this periodic physical visits for monitoring [8]. In this pandemic period, a pilot study concluded that telemonitoring has proved to be beneficial in dealing with surgical and non- surgical dental patients [9]. Basically, it is used for monitoring patients at distance.

\section{TELEDENTISTRY AND COVID 19}

Our healthcare system comprising of one of the most important part the dentistry, has become severely compromised due to current pandemic situation of COVID-19. The dental practice has to be redefined with new screening technologies. Incorporation of teledentistry into routine dental procedure is the need of the hour. Teledentistry can be used in different aspects to reduce the contact of healthy or uninfected persons with the infected persons. The basis of health care system lies in the well-being and welfare of the health workers and professionals. To combat this increasing incidences of Covid-19, this telecommunication can play pivotal role in maintaining social distancing. The 'stay home' propaganda can only be followed if individuals can resume their professional work at home and in case of dentistry, this can be achieved by considering and proper implementing this tele technology. To resist the risk of cross infection new approaches such as teledentistry will help the dentists to assist patients at various levels.

\section{CHALLENGES IN IMPLEMENTATION OF TELEDENTISTRY}

One of the major barrier is that every state has its own different licensure requirements for the application of telemedicine and teledentistry. Along with numerous advantages, use of teledentistry is very crucial as it can also lead to misdiagnosis or medical error due to any existing technical errors during data transmission. In addition to this, privacy and security of patients details must be maintained in the cyberspace $[10,11]$. Obviously, humans cannot be replaced by the technology so it would be a misbelieve that dental care providers can be reinstated by teledentistry. The 'cost factor' is also an addition to the hurdles in teledentistry.

\section{CONCLUSION}

Teleconsultations has not only increased patient compliance but also established a strong relationship between patients and doctors. Keeping in account the current situation, the implementation of teledentistry will not only bridge the gap between patients and consultants but also provides safety in all aspects. Human- to- human transmissions and nosocomial spread can be diminuted with the application of this tele- technology. This teledentistry consultations can be continued after this dreadful phase of pandemic is over. The establishment of teledentistry should be considered as prime means of connectivity to 
overcome this crisis. The need of the current situation is to maintain a balance between safety of health care professionals along with providing adequate dental care.

\section{FUTURE RECOMMENDATIONS}

Dentists should be well-versed with the concept and technology of teledentistry. All collaborators are urged to collaborate to impede effective and evidence-based usage of teledentistry across the current pandemic and potential outbreaks. Criteria for screening suspicious activities should be developed and the conditions for compensation, interstate licensing and data privacy issues should be revised.

\section{REFERENCES}

1. Centers for Disease Control and Prevention, Interim Infection Prevention and Control Guidance for Dental Settings during the COVID19 Response, Centers for Disease Control and Prevention, Atlanta, GA, USA, 2019, https://www.cdc.gov/coronavirus/2019ncov/hcp/dental-settings.html.

2. Rocca, M. A., Kudryk, V. L., Pajak, J. C., \& Morris, T. (1999). The evolution of a teledentistry system within the Department of Defense. In Proceedings of the AMIA Symposium (p. 921). American Medical Informatics Association.

3. Spivack, E. (2020). Teledentistry: remote observation of patients with special needs. General dentistry, 68(3), 66-70.

4. Tynan, A., Deeth, L., McKenzie, D., Bourke, C., Stenhouse, S., Pitt, J., \& Linneman, H. (2018). Integrated approach to oral health in aged care facilities using oral health practitioners and teledentistry in rural Queensland. Australian Journal of Rural Health, 26(4), 290-294.

5. AlShaya, M. S., Assery, M. K., \& Pani, S. C. (2020). Reliability of mobile phone teledentistry in dental diagnosis and treatment planning in mixed dentition. Journal of telemedicine and telecare, 26(1-2), 45-52.

6. Kohara, E. K., Abdala, C. G., Novaes, T. F., Braga, M. M., Haddad, A. E., \& Mendes, F. M. (2018). Is it feasible to use smartphone images to perform telediagnosis of different stages of occlusal caries lesions?. PloS one, 13(9), e0202116.

7. Haron, N., Zain, R. B., Ramanathan, A., Abraham, M. T., Liew, C. S., Ng, K. G., ... \& Cheong, S. C. (2020). m-Health for early detection of oral cancer in low-and middle-income countries. Telemedicine and e-Health, 26(3), 278285.

8. Mariño, R., \& Ghanim, A. (2013). Teledentistry: a systematic review of the literature. Journal of Telemedicine and Telecare, 19(4), 179-183.

9. Giudice, A., Barone, S., Muraca, D., Averta, F., Diodati, F., Antonelli, A., \& Fortunato, L. (2020). Can teledentistry improve the monitoring of patients during the Covid-19 dissemination? A descriptive pilot study. International journal of environmental research and public health, 17(10), 3399.

10. Golder, D. T., \& Brennan, K. A. (2000). Practicing dentistry in the age of telemedicine. The Journal of the American Dental Association, 131(6), 734-744.

11. Biegel, S. (2000). Virtual health care: unresolved legal issues. Journal of the California Dental Association, 28(2), 128-132. 